03

\title{
Управление течением в гиперзвуковом пограничном слое
}

\author{
(С) И.И. Липатов ${ }^{1,2}$, Ван Кхьем Фам ${ }^{1, \uparrow}$ \\ ${ }^{1}$ Московский физико-технический институт (Национальный исследовательский университет), Долгопрудный, \\ Московская обл., Россия \\ ${ }^{2}$ Центральный аэрогидродинамический институт им. проф. Н.Е. Жуковского, Жуковский, Московская обл., Россия \\ E-mail: igor_lipatov@mail.ru \\ ฯE-mail: van.fam@phystech.edu
}

Поступило в Редакцию 7 июня 2019г.

В окончательной редакции 17 марта 2020г.

Принято к публикации 17 марта 2020г.

Рассмотрен метод управления течением в двумерном гиперзвуковом пограничном слое в условиях сильного взаимодействия. Показан эффект блокирования процесса передачи возмущений вверх по потоку за счет уменьшения скорости распространения возмущений при уменьшении температурного фактора участка поверхности.

Ключевые слова: асимптотическая теория вязкого газа, управление течением, распространение возмущений, вязко-невязкое взаимодействие.

DOI: 10.21883/PJTF.2020.11.49500.17913

Существуют различные подходы к управлению течением в пограничном слое. Целью управления является увеличение числа Рейнольдса ламинарно-турбулентного перехода, устранение отрыва и т.д. В большинстве методов управления используются подходы, связанные с добавлением энергии в пограничный слой путем выдува струй или электрических разрядов и др. Некоторые из таких методов описаны в работах [1-3].

В настоящей работе управление направлено на устранение или уменьшение эффектов распространения возмущений в гиперзвуковых пограничных слоях. Это в ряде ситуаций оказывается важным для эффективной работы аэродинамических органов управления, хотя также может применяться для управления отрывом или переходом.

Распространение возмущений в пограничных слоях связано с процессами конвекции и диффузии [4]. Анализ системы уравнений пограничного слоя $[4,5]$ показал, что характеристики этой системы уравнений - линии, нормальные к обтекаемой поверхности. Система характеристик и субхарактеристик позволяет анализировать зоны зависимости и влияния. Из-за вязкости возникает область дозвукового обтекания, поэтому существует возможность передачи возмущений вверх по потоку, обусловленная волновыми процессами. Экспериментальные данные, подтверждающие это, приведены в [6].

Рассмотрены стационарные процессы в гиперзвуковом пограничном слое в условиях сильного вязко-невязкого взаимодействия. Из-за взаимодействия распределение давления заранее неизвестно, и оно должно определяться в процессе решения задачи. В этом случае существует дополнительный механизм передачи возмущений, связанный с распространением волн давления. Поскольку в пограничном слое существует область дозвукового течения вблизи поверхности обтекаемого тела, эффект передачи возмущений вверх по потоку имеет место.

Передача возмущений вверх по потоку может привести к изменению распределения давления и других параметров на поверхности обтекаемого тела. Этот вывод относится как к стационарным, так и к нестационарным течениям. Известны эксперименты, в которых влияние давления в донной области приводило к потере устойчивости конусов, обтекаемых сверхзвуковым потоком.

Анализ распространения возмущений в пограничном слое при вязко-невязком взаимодействии позволил определять субхарактеристические поверхности, отделяющие области докритического (дозвукового в среднем) и закритического (сверхзвукового в среднем) течения в пограничном слое. В настоящей работе предложен метод управления течением с помощью охлаждения участка пластины. Показано, что скорость распространения возмущений уменьшается до нуля при уменьшении температурного фактора до нуля. Таким образом, если передача возмущений вверх по потоку на части поверхности или на всей поверхности отсутствует, это может привести к изменению распределения давления и других параметров на поверхности обтекаемого тела.

Рассматривается обтекание плоской поверхности, расположенной под нулевым углом атаки к набегающему гиперзвуковому потоку вязкого теплопроводного газа. При режиме сильного вязко-невязкого взаимодействия имеют место следующие соотношения:

$$
\mathrm{M}_{\infty} \rightarrow \infty, \quad \mathrm{M}_{\infty} \tau \rightarrow \infty
$$

где $\mathbf{M}_{\infty}$ - число Маха набегающего потока, $\tau$ - безразмерная толщина ламинарного пограничного слоя; также предполагается, что число Рейнольдса $\operatorname{Re}_{0}=\rho_{\infty} u_{\infty} l_{\infty} / \mu_{0}$ велико, но не превосходит критического значения, при котором возникает ламинарно- 
турбулентный переход. Здесь $\rho_{\infty}, u_{\infty}-$ плотность и скорость набегающего потока, $l_{\infty}-$ характерная длина, $\mu_{0}$ - вязкость газа.

Согласно теории сильного вязко-невязкого взаимодействия, область возмущенного течения вблизи обтекаемого тела разделяется на две подобласти: невязкое течение и пограничный слой $[7,8]$.

Для невязкого течения функции течения могут представляться в асимптотическом виде

$$
\begin{gathered}
x=x_{1}, \quad y=\tau y_{1}, \\
u(x, y, \tau)=1+\ldots, v(x, y, \tau)=v_{1}\left(x_{1}, y_{1}\right)+\ldots, \\
p(x, y, \tau)=\tau^{2} p_{1}\left(x_{1}, y_{1}\right)+\ldots, \\
\rho(x, y, \tau)=\rho_{1}\left(x_{1}, y_{1}\right)+\ldots,
\end{gathered}
$$

где $u(x, y, \tau), v(x, y, \tau)$ - продольная и поперечная компоненты поля скорости, $p(x, y, \tau)$ - возмущение давления, $\rho(x, y, \tau)$ - плотность газа.

Подставляя (2) в систему уравнений Навье-Стокса и учитывая предельный переход (1), получим следующую систему уравнений:

$$
\left\{\begin{array}{l}
\frac{\partial \rho_{1}}{\partial x_{1}}+\frac{\partial \rho_{1} v_{1}}{\partial y_{1}}=0 \\
\frac{\partial v_{1}}{\partial x_{1}}+v_{1} \frac{\partial v_{1}}{\partial y_{1}}+\frac{1}{\rho_{1}} \frac{\partial p_{1}}{\partial y_{1}}=0 \\
\frac{\partial}{\partial x_{1}}\left(\frac{p_{1}}{p_{1}^{\gamma}}\right)+v_{1} \frac{\partial}{\partial y_{1}}\left(\frac{p_{1}}{p_{1}^{\gamma}}\right)=0
\end{array}\right.
$$

с граничными условиями на ударной волне

$$
\begin{array}{cl}
y_{1}=g_{1}\left(x_{1}\right), & \rho_{1}=\frac{\gamma+1}{\gamma-1}, \\
p_{1}=\frac{(\gamma+1) v_{1}^{2}}{2}, & v_{1}=\frac{2}{\gamma+1} \frac{\partial g_{1}}{\partial x_{1}}
\end{array}
$$

и на внешней границе пограничного слоя

$$
y_{1}=\delta_{1}\left(x_{1}\right), \quad v_{1}=\frac{2}{\gamma+1} \frac{\partial \delta_{1}}{\partial x_{1}},
$$

где $\gamma=1.4-$ показатель адиабаты.

Для получения связи между толщиной пограничного слоя $\delta_{1}$ и возмущением давления $p_{1}\left(x_{1}\right)$ использована формула Ньютона $p_{1}=(\gamma+1) v_{1}^{2} / 2$.

Эта формула может быть строго выведена из приведенной выше системы уравнений в пределе при больших числах Маха (что предполагается в настоящей работе) и при отношении удельных теплоемкостей, стремящемся к единице. Последнее предположение в работе не используется, поскольку предполагается, что приближенная формула Ньютона описывает возмущенное течение с достаточной точностью.

Для пограничного слоя характерны следующие асимптотические разложения и представления координат:

$$
x=x_{1}, \quad y=\tau y_{1},
$$

$$
\begin{gathered}
u(x, y, \tau)=u_{2}\left(x_{1}, y_{1}\right)+\ldots, \\
v(x, y, \tau)=\tau v_{2}\left(x_{1}, y_{1}\right)+\ldots, \\
p(x, y, \tau)=\tau^{2} p_{2}\left(x_{1}\right)+\ldots, \\
\rho(x, y, \tau)=\tau^{2} \rho_{2}\left(x_{1}, y_{1}\right)+\ldots, \\
H(x, y, \tau)=H_{2}\left(x_{1}, y_{1}\right)+\ldots,
\end{gathered}
$$

где $H(x, y, \tau)$ - полная энтальпия.

Подставляя (3) в систему уравнений Навье-Стокса и учитывая предельный переход (1), получим систему уравнений стационарного пограничного слоя. С помощью замены переменных (замена Дородницына-Лиза)

$$
\begin{gathered}
X=x_{1}, \quad Y=\sqrt{\frac{2 \gamma C_{0}}{\gamma-1}} x_{1}^{-1 / 4} \int_{0}^{y_{1}} R d y_{1}, \\
u_{2}=\frac{\partial F}{\partial Y}, \quad p_{2}=x_{1}^{-1 / 2} P, \quad \rho_{2}=x_{1}^{-1 / 2} R, \\
C_{0}=P_{X=0}, \quad G=H_{2}, \quad Q=G-U^{2}
\end{gathered}
$$

(где $C_{0}$ - давление на передней кромке, $F-$ функция тока, $U-$ продольная скорость, $G$ - энтальпия, $P-$ давление, $R$ - плотность) получим следующую систему уравнений:

$$
\left\{\begin{array}{l}
X\left(U \frac{\partial U}{\partial X}-\frac{\partial F}{\partial X} \frac{\partial U}{\partial Y}\right)-\frac{F}{4} \frac{\partial U}{\partial Y}+\beta \frac{\gamma-1}{4 \gamma} Q=\frac{P}{C_{0}} \frac{\partial^{2} U}{\partial Y^{2}} \\
X\left(U \frac{\partial G}{\partial X}-\frac{\partial F}{\partial X} \frac{\partial G}{\partial Y}\right)-\frac{F}{4} \frac{\partial G}{\partial Y}=\frac{P}{C_{0}} \frac{\partial^{2} G}{\partial Y^{2}} \\
U=\frac{\partial F}{\partial Y}
\end{array}\right.
$$$$
\Delta=\sqrt{\frac{(\gamma-1) C_{0}}{2 \gamma P^{2}}} \int_{0}^{\infty} Q d Y, \quad P=\frac{(\gamma+1)}{2}\left(\frac{3 \Delta}{4}+X \frac{\partial \Delta}{\partial X}\right)^{2}
$$

с граничными условиями

$$
\begin{gathered}
U=F=0, \quad G=g_{w}, \quad Y=0, \\
U=G=1, \quad Y=\infty,
\end{gathered}
$$

где $\gamma=1.4, \beta=-1+\frac{2 X}{P} \frac{\partial P}{\partial X}, \Delta-$ толщина вытеснения пограничного слоя, $P-$ поле давления, $g_{w}-$ температурный фактор.

Для определения единственного решения приходится задать дополнительное условие на некоторой линии ниже по течению от передней кромки, например донное давление на задней кромке [9]. Появление дополнительного условия связано с возможным распространением возмущений вверх по потоку и их влиянием на течение в пограничном слое.

Использован метод конечных разностей второго порядка точности по $X$ и четвертого порядка точности по $Y ; \Delta$ вычисляется с помощью формулы Симпсона четвертого порядка точности. Для данной задачи шаги по $X$ и $Y$ равны 0.025 и 0.1 и обеспечивают приемлемую 

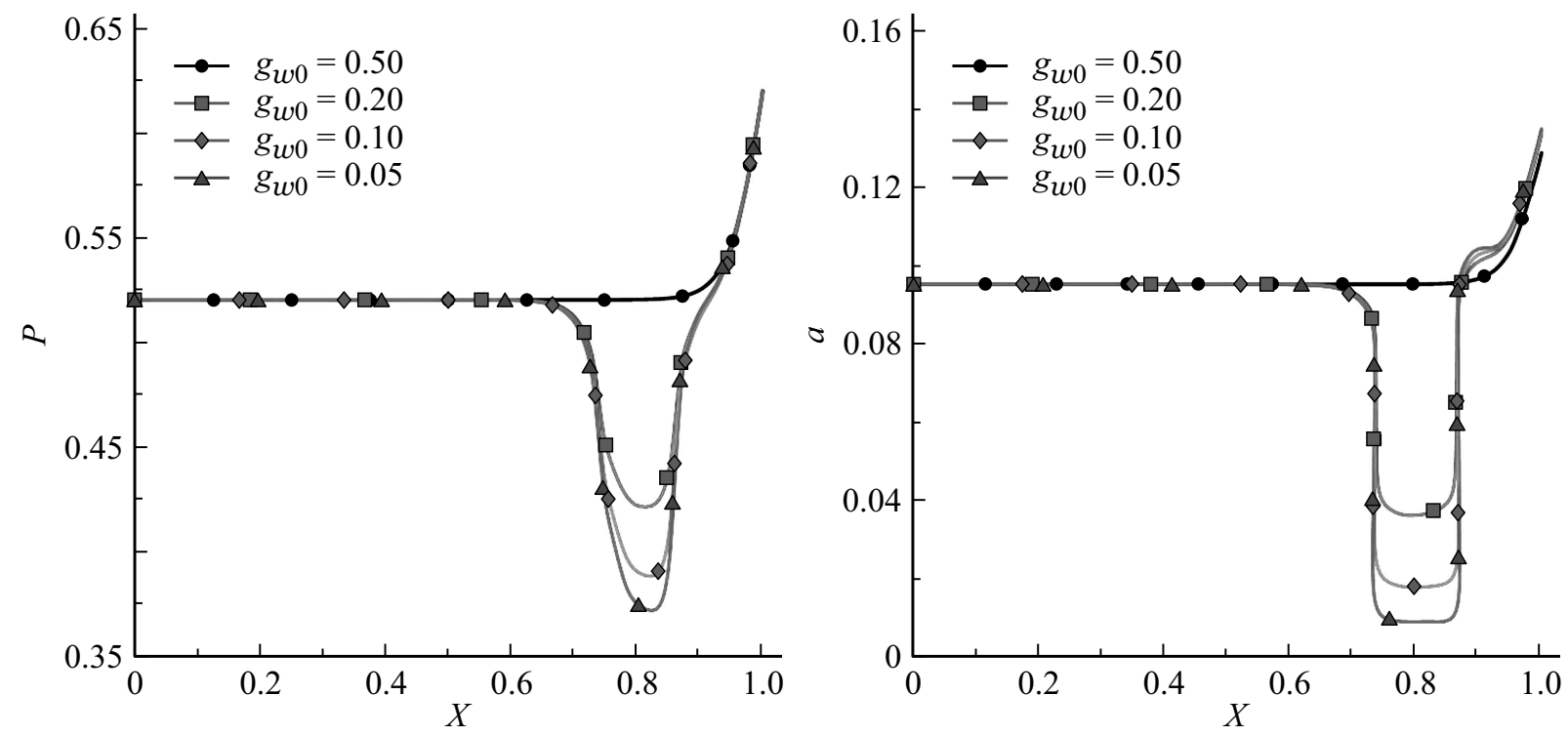

Рис. 1. Распределение давления и скорость распространения возмущений вверх по потоку при уменьшении температурного фактора $g_{w 0}$ участка поверхности $X=0.75-0.85$ при условии $P_{X=1}=P_{X=0}+0.1$ и $g_{w}=0.5$.
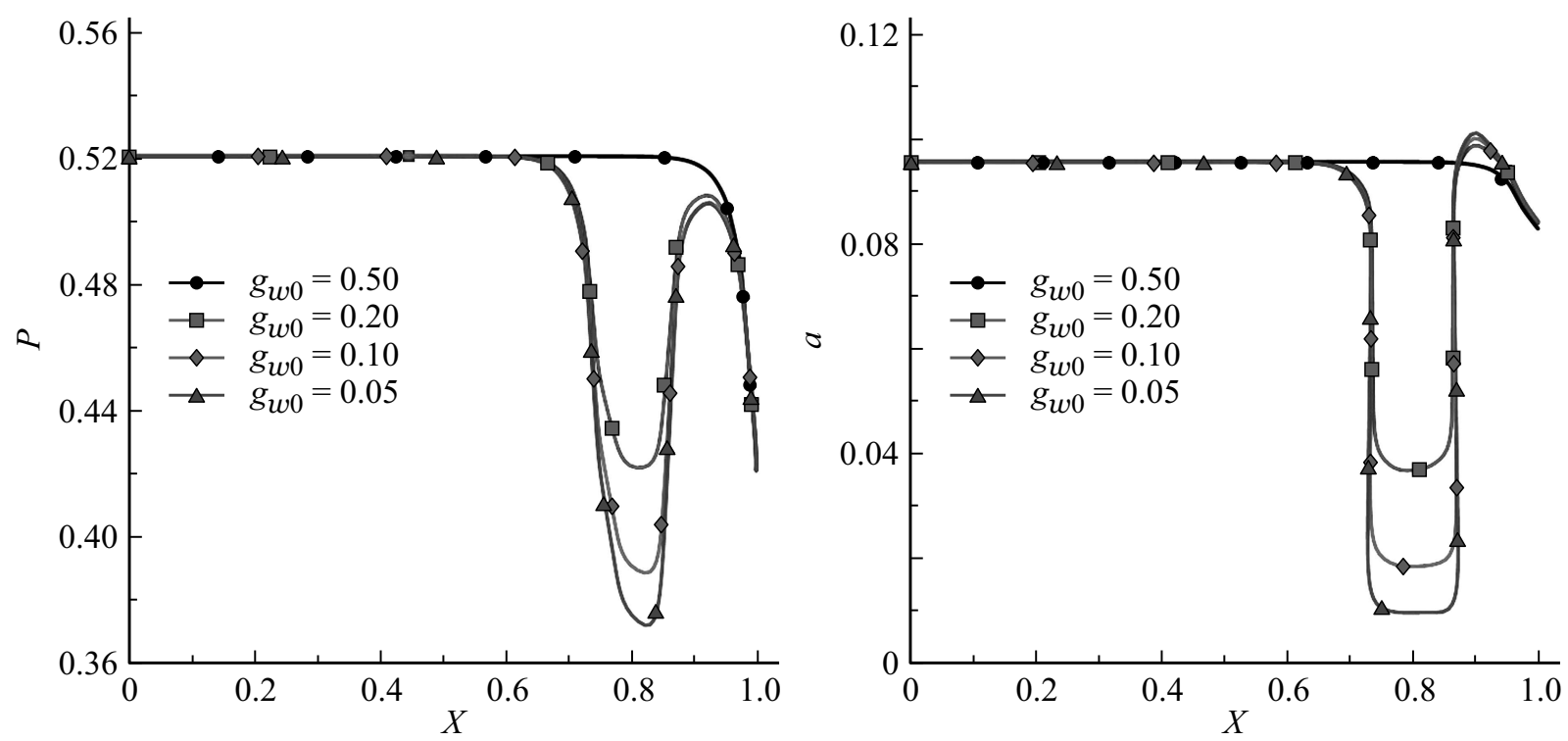

Рис. 2. Распределение давления и скорость распространения возмущений вверх по потоку при уменьшении температурного фактора $g_{w 0}$ участка поверхности $X=0.75-0.85$ при условии $P_{X=1}=P_{X=0}-0.1$ и $g_{w}=0.5$.

точность. Из-за вязко-невязкого взаимодействия давление заранее неизвестно, мы знаем значение давления только при $X=0$ и $X=1$, поле давления определяется методом глобальной итерации $[10,11]$ с помощью решения дополнительного обыкновенного дифференциального уравнения второго порядка.

В качестве примера заданы величина донного давления $P_{X=1}=P_{X=0} \pm 0.1$ и температурный фактор $g_{w}=0.5$. Рассмотрено влияние уменьшения температурного фактора на участке поверхности $X=0.75-0.85$ на распределение давления и скорость распространения возмущений вверх по потоку. Этот выбор достаточно произволен, хотя для изменения моментных характеристик существенны изменения давления на большей части обтекаемого тела. Давление $P_{X=0}$ определяется исходя из автомодельной задачи, которая получена из системы уравнений (4) при $X=0$. Скорость распространения возмущений вверх по потоку определяется через модифицированный интеграл Пирсона [12], если известны профили скорости и энтальпии:

$$
\frac{\gamma-1}{2} \int_{0}^{\infty} \frac{\left(G-U^{2}\right)^{2}}{(U+a)^{2}} d Y-\int_{0}^{\infty}\left(G-U^{2}\right) d Y=0
$$


Температурный фактор $g_{w}$ равен отношению температуры поверхности к температуре торможения. Охлаждение поверхности приводит к уменьшению толщины пограничного слоя и соответствующему относительному уменьшению толщины области дозвукового течения. В результате уменьшается скорость распространения возмущений вверх по потоку. Численные результаты (рис. 1,2 ) показали, что при стремлении температурного фактора $g_{w}$ к нулю скорость распространения возмущений вверх по потоку также стремится к нулю.

Таким образом, установлено, что при охлаждении участка поверхности наблюдалось уменьшение скорости распространения возмущений вверх по потоку. При температурном факторе, стремящемся к нулю, скорость распространения возмущений также стремится к нулю. Это эффект блокировки распространения возмущений вверх по потоку. Поэтому можно охлаждать участок поверхности для управления течением в гиперзвуковом пограничном слое. Этот эффект может иметь существенное значение для задач прикладной аэродинамики, равно как и для задач гиперзвуковых течений.

\section{Конфликт интересов}

Авторы заявляют, что у них нет конфликта интересов.

\section{Список литературы}

[1] Панина А.В., Косинов А.Д., Ермолаев Ю.Г., Горев В.Н., Семёнов Н.В. // Теплофизика и аэромеханика. 2014. Т. 21. № 1. C. 3-13.

[2] Гапонов С.A., Терехова Н.М. // Теплофизика высоких температур. 2017. Т. 55. В. 6. С. 733-741.

[3] Баранов С.А., Киселёв А.Ф., Моралев И.А., Сбоев Д.С., Толкачёв С.Н., Чернышев С.Л. // ДАН. 2019. Т. 486. № 6. C. $668-672$.

[4] Нейланд В.Я., Боголепов В.В., Дудин Г.Н., Липатов И.И. Асимптотическая теория сверхзвуковых течений вязкого газа. М.: Физматлит, 2003. 456 с.

[5] Wang K.C. // J. Fluid Mech. 1971. V. 48. N 2. P. 397-404.

[6] Lighthill M.J. // Proc. Roy. Soc. A. 1953. V. 217. N 1131. P. 478-507.

[7] Lipatov I.I., Cho T.A. // Adv. Appl. Math. 2014. N 4. P. 635 640.

[8] Липатов И.И., Дубинский С.В. // Письма в ЖТФ. 2008. T. 34. B. 2. C. $32-38$.

[9] Нейлад В.Я. // Изв. АН СССР. Механика жидкости и газа. 1970. № 4. С. 40-49.

[10] Дудин Г.Н., Ледовский А.В. // Учен. зап. ЦАГИ. 2011. T. 42. № 2. C. 11-25.

[11] Башкин В.А., Дудин Г.Н. Пространственные гиперзвуковые течения вязкого газа. М.: Физматлит, 2000. 289 с.

[12] Pearson H., Holliday J.B., Smith S.F. // J. Roy. Aeron. Soc. 1958. V. 62. N 574. P. 746-751. 